\title{
Tetracycline and the ecdysis level in cultures of the dinoflagellate Prorocentrum minimum
}

\author{
Mariia Berdieva, Sofia Pechkovskaya and Olga Matantseva \\ Institute of Cytology, Russian Academy of Sciences, 194064 St. Petersburg, Russia
}

| Submitted April 17, 2020|Accepted May 11, 2020|

\begin{abstract}
Summary
Many dinoflagellate species are sensitive to mechanical disturbances, for instance, to mixing, which hampers effective continuous culturing of these organisms. Such sensitivity may be linked to the induction of ecdysis, a process of the cell covering rearrangement, followed by temporary quiescence of the dinoflagellate cells. Ecdysis can be induced in a significant fraction of a dinoflagellate population by unfavorable environmental conditions and is often involved in the formation of their cysts. Previously it was demonstrated that the bacteriostatic antibiotic tetracycline, which is widely used against bacteria in aquaculture, decreased the natural ecdysis rate in the culture of the dinoflagellate Prorocentrum minimum (Pavillard) Schiller, 1933. In this study, we tested the ability of this compound to lower the rate of ecdysis induced by mechanical stimuli. We showed that mechanical disturbances associated with centrifugation, stirring, and shaking equally induced ecdysis in the tetracyclinetreated and control cultures. Thus, tetracycline did not make dinoflagellates less susceptible to the mechanical stressors and cannot be used to surpass their sensitivity to mixing during continuous culturing.
\end{abstract}

Key words: ecdysis, Prorocentrum minimum, tetracycline

\section{Introduction}

Dinoflagellates are single-celled eukaryotic organisms representing important primary producers and grazers in the marine environment (Hackett et al., 2004). They are infamously known to produce potent toxins, such as saxitoxin, brevetoxin and okadaic acid, which can be accumulated by marine organisms to the levels threatening human health. The events of the rapid dinoflagellate proliferation called harmful blooms are especially dangerous in this respect, since they are associated with the intensive toxin production (Hallegraeff, 1993; Wang, 2008). All these features make dinoflagellates one of the threats to aquaculture maintaining. Potent toxins synthesized by them can be accumulated in tissues of clams and fish thus threatening consumers of cultured organisms (Shumway, 1990; Matsuyama, 2012; Brown et al., 2020). Therefore, monitoring of fluctuations in the number of dinoflagellate cells in water is an important challenge for aquaculture management.

Dinoflagellates possess many peculiar traits of the cell structure and physiology important for 
their activity, including bloom formation, in the environment. One of such traits is the special organization of a cell covering, called amphiesma, consisting of a plasma membrane and membrane vesicles beneath it. In armored species, amphiesmal vesicles contain thecal plates made of cellulose. In the course of a dinoflagellate cell life cycle, amphiesma can be subjected to a substantial rearrangement termed ecdysis (Morrill and Loeblich, 1983; Morrill, 1984; Pozdnyakov and Skarlato, 2012).

During ecdysis, amphiesmal vesicles fuse to form two continuous membranes (outer and inner amphiesmal vesicle membranes) beneath the plasma membrane. Then the plasma membrane, outer amphiesmal vesicle membrane and thecal plates (if present) are discarded, and the inner amphiesmal vesicle membrane becomes the new plasma membrane. Subsequently, new amphiesmal vesicles appear and mature to restore the full amphiesma (Höhfeld and Melkonian, 1992; Pozdnyakov and Skarlato, 2012 and references therein; Matantseva et al., 2020). The rearrangement of a dinoflagellate cell covering is linked to the formation of their cysts and can be induced by various external triggers, e.g. mechanical disturbances, changes in temperature and light conditions (Bravo and Figueroa, 2014).

Whereas many triggers have been reported to induce ecdysis, it is less clear whether some factors can hinder the initiation and progression of this process. Previously it was demonstrated that the bacteriostatic antibiotic tetracycline decreased the natural background level of ecdysis in the laboratory culture of the dinoflagellates Prorocentrum minimum (syn. Prorocentrum cordatum (Ostenfeld) Dodge, 1975) (Matantseva et al., 2020). The finding that a chemical agent can suppress the dinoflagellate ecdysis initiated by unknown internal or external stimuli further raises a question whether the same agent can affect ecdysis triggered by known external stressors. Mechanical impacts attract special attention since they are widely distributed in both natural (tidal and wind/wave activity) and laboratory (mixing during culturing) habitats and thus are highly significant for the dinoflagellate population dynamics (Pollingher and Zemel, 1981; Berdalet, 1992; Wyatt and Jenkinson, 1997; Smayda, 2010; van de Waal et al., 2014). In turn, tetracycline and its derivatives are antibiotics commonly used in aquaculture worldwide (Alderman and Hastings, 1998; Sapkota et al., 2008; Guidi et al., 2018; Lulijwa et al., 2020).
In this study, we tested experimentally the hypothesis that the application of tetracycline to the culture of $P$. minimum can affect the rate of ecdysis induced by mechanical disturbances. Due to the huge impact of dinoflagellates on mariculture, this information could be of practical value.

\section{Material and methods}

\section{CUlture MATERIAL AND GROWTH CONDITIONS}

The clonal culture of Prorocentrum minimum, strain CCAP 1136/16, was maintained in artificial seawater-based f/2 medium (Guillard and Ryther, 1962) containing no silicate at salinity of 25 . The cells were grown under a $12 \mathrm{~h} / 12 \mathrm{~h}$ light/dark cycle at $100 \mu \mathrm{mol}$ photons $\mathrm{m}^{-2} \mathrm{~s}^{-1}$ and $18{ }^{\circ} \mathrm{C}$.

For experiments, cells were inoculated into Erlenmeyer glass flasks with fresh $\mathrm{f} / 2$ medium at a cell concentration of $40 \times 10^{3}$ cells ml-1 and then allowed to grow for four days to reach the exponential growth phase. All experiments were performed on the fourth day.

\section{EXPERIMENTAL PROCEDURES}

In order to estimate the effect of tetracycline on the ecdysis rate, we applied several different types of mechanical disturbance to samples incubated with the antibiotic and without it. Stock solution of tetracycline (B1418, ChemCruz, Santa Cruz Biotechnology, Dallas, Texas, USA) was added into the samples to a final concentration of $10 \mu \mathrm{g} \mathrm{ml}^{-1}$.

Ecdysis was triggered experimentally by the following treatments: shaking, mixing, and centrifugation. For the first treatment, $10 \mathrm{ml}$ of the culture were placed into Erlenmeyer glass flasks with the bottom diameter $65 \mathrm{~mm}$. These samples were shaken for $24 \mathrm{~h}$ at $200 \mathrm{rpm}$ in the environmental shaker-incubator ES-20 (Biosan, Riga, Latvia). The second treatment included mixing of $10-\mathrm{ml}$ samples in Erlenmeyer glass flasks with the bottom diameter $65 \mathrm{~mm}$ using magnetic stirrer MS-3000 (Biosan, Riga, Latvia), the relative speed 2.5, and a $15 \mathrm{~mm}$ magnet for $24 \mathrm{~h}$. For the third treatment, 1 -ml samples were centrifuged for $5 \mathrm{~min}$ at $10000 \mathrm{~g}$, and then cells were resuspended and incubated for 4 $\mathrm{h}$ before the determination of the ecdysis rate. The natural ecdysis level in the culture and the effect of tetracycline on it were examined by incubating 10 
$\mathrm{ml}$ of the experimental culture in the normal media and in the media containing tetracycline $\left(10 \mu \mathrm{g} \mathrm{ml}^{-1}\right)$ in Erlenmeyer glass flasks for $24 \mathrm{~h}$ at the standard conditions. All experiments were conducted in 3-4 replicates.

\section{ANALYTICAL PROCEDURES}

To determine the ecdysis level, cells and empty separated thecal plates were counted in samples using a Fuchs-Rosenthal counting chamber and a light microscope. Before counting, all samples were vortexed to immobilize cells. At least 200 cells per sample were counted. The following formula was applied to estimate the ecdysis rate:

$$
E_{\text {thecae }}=\frac{N_{\text {thecae }} / 2}{N_{\text {cells }}}
$$

where $N_{\text {thecae }}-$ number of empty thecal plates, $N_{\text {cells }}-$ number of cells.

\section{Results}

The addition of tetracycline to the culture media led to the lower background level of ecdysis in the cultures of Prorocentrum minimum compared to the control cultures (paired t-test, $n=4, p=0.0246$ ) (Fig. 1, A). The mean background level of ecdysis in nontreated cultures constituted $0.062 \pm 0.013$, whereas in the tetracycline-treated cultures it was $0.031 \pm 0.007$.

Various mechanical disturbances, including centrifugation at $10000 \mathrm{~g}$ for $5 \mathrm{~min}$, shaking on a rotation shaker at a speed of $200 \mathrm{rpm}$ for $24 \mathrm{~h}$, and mixing using a magnetic stirrer at the relative speed of 2.5 for $24 \mathrm{~h}$, induced ecdysis in the $P$. minimum cultures. The level of ecdysis in $4 \mathrm{~h}$ after centrifugation and immediately after the completion of shaking and stirring was, on overage, 0.4. No statistically significant differences were found between the level of ecdysis in cultures growing on normal and tetracycline-containing media if cultures were subjected to centrifugation (Fig. 1, B), shaking (Fig. 1, C), and stirring (Fig. 1, D) (paired t-test, $n=3-4, p>0.05$ ). It must be noted that albeit no statistically significant effect of tetracycline was revealed in the case of shaking at a chosen significance level, the mean rate of ecdysis induced by this disturbance was nearly two times lower in the tetracycline-treated cultures (Fig. 1, C).

\section{Discussion}

The growing demand of humanity in food accompanied by a decrease in natural catch have led to the development of aquaculture worldwide (Bostock et al., 2010; Merino et al., 2012). Culturing of aquatic organisms is associated with the outburst of infectious diseases among them (Lafferty et al., 2015). One of the main approaches to cope with this problem is the treatment of aquaculture with antibiotics, in particular, tetracycline and its derivatives (Alderman and Hastings, 1998; Sapkota et al., 2008; Guidi et al., 2018; Lulijwa et al., 2020). The elevated concentration of tetracycline in the regions of aquaculture may influence different components of ecosystems. Therefore, the effects of tetracycline-like antibiotics on organisms other than bacteria should be investigated.

The concentration of tetracycline chosen in this experimental study corresponds to the concentration used in Matantseva et al. (2020) for which the effect of tetracycline on the general ecdysis level in P. minimum culture was demonstrated for the first time. This concentration is in the range of the maximal tetracycline dosages applied to aquaculture (e.g. Alderman and Hastings, 1998; Armstrong et al., 2005). Thus, this concentration of the antibiotic is the maximum level that dinoflagellates can be exposed to in the natural habitats. To study the effect of tetracycline on the rate of ecdysis triggered by external stressors, it was necessary to induce the ecdysis exceeding the natural ecdysis level in the culture. We tested several variants of mechanical treatment in the study. The experiments were designed according to the results of the previous work (Matantseva et al., 2020) or preliminary testing. For shaking and stirring, settings have been chosen at the minimum level which stably caused ecdysis exceeding the natural rate. Centrifugation has been applied in the previous study and has appeared to be one of the potent stimuli inducing ecdysis in P. minimum (Matantseva et al., 2020). While we cannot estimate the intensity of mechanical impact precisely and match the strength of the experimental treatments to natural stressors, such stressor-induced ecdysis appears to be an appropriate model. In the absence of data on the actual trigger of ecdysis during mechanical disturbances both in natural and experimental systems, different types of treatments known to trigger ecdysis may be applied to elucidate the plausible role of tetracycline.

Herein we confirmed the effect of tetracycline on the natural rate of ecdysis in the dinoflagellate $P$. 
A

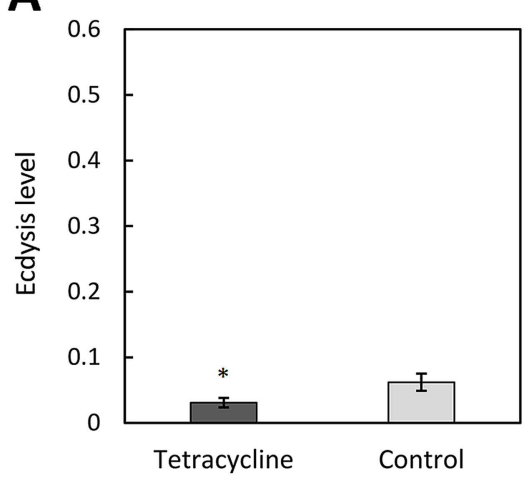

C

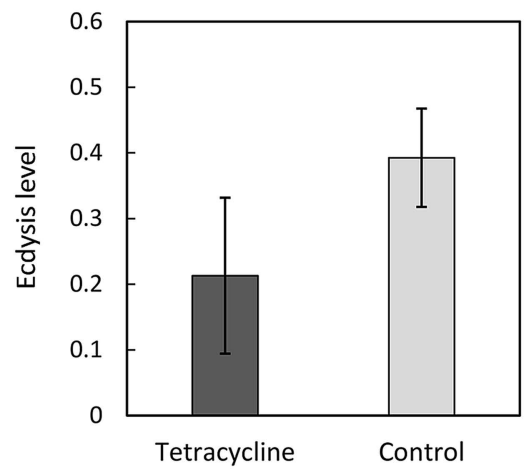

B

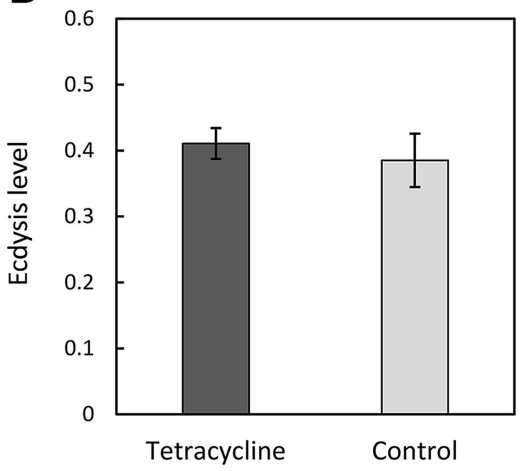

D

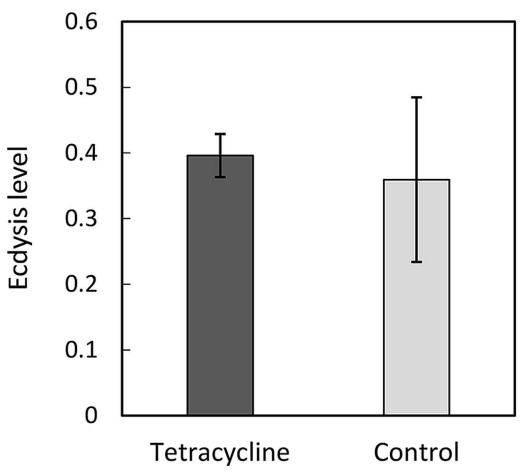

Fig. 1. Effect of tetracycline on the level of ecdysis induced by various mechanical treatments in P. minimum culture. A - No mechanical treatment; B - centrifugation; C - shaking; D - stirring. Asterisk indicates statistically significant difference (paired t-test, $n=4, p=0.0246$ ).

minimum previously revealed by Matantseva et al. (2020). This peculiar fact indicates that tetracycline may represent an agent protecting dinoflagellate cells from stressors initiating ecdysis and cyst production. This phenomenon might be relevant for the regions of aquaculture and for the continuous cultures of these organisms.

Population dynamics and activity of dinoflagellates both in natural habitats and in culture is linked to the production of cysts and temporary quiescence during the process of ecdysis which are often caused by mechanical disturbance. Our experiments demonstrated that tetracycline did not reduce the level of ecdysis if cultures were subjected to significant mechanical impacts including centrifugation, shaking, and stirring. Thus, it cannot be used to improve the efficiency of continuous culturing of dinoflagellates requiring mixing and unlikely to be important for the plankton-benthos shifts associated with wind and wave activity in natural waters containing this antibiotic. It was shown that the membrane fluidity in dinoflagellates
Lingulodinium polyedra is affected by fluid shear stress (Mallipattu et al., 2002). This probably represents a mechanism behind the sensitivity of dinoflagellates to mechanical stressors, although special investigations are required to solve the issue of what exactly triggers ecdysis. Apparently, tetracycline does not stabilize the membrane directly or indirectly to make it resistant to the treatments applied. However, we should mention a decrease (albeit statistically insignificant) in the mean rate of ecdysis in the tetracycline-treated cultures exposed to shaking relative to the control. Although this decrease could arise from random fluctuations in the ecdysis level, the role of tetracycline in the ecdysis suppression or a possible delay in the shedding of thecal plates cannot be completely ruled out and merit additional investigation.

The background level of ecdysis in cultures that were not subjected to centrifugation, shaking or stirring, can be explained in two ways. First, culture agitation caused by pipetting and thus inevitable during the experimental work could 
represent a mechanical stressor of low intensity triggering ecdysis in a small fraction of a population. Previously it was shown that centrifugation at $500 \mathrm{~g}$ representing a disturbance of low intensity induced ecdysis in a smaller fraction of the dinoflagellate population compared to centrifugation at $5000 \mathrm{~g}$ and $10000 \mathrm{~g}$ (Matantseva et al., 2020). It is possible that tetracycline can protect dinoflagellate cells from the membrane damage only in the case of weak shear stress, whereas in the case of more intensive impact its protective effect is insufficient. Second, it cannot be excluded that P. minimum cells enter ecdysis not only because of the fluid shear stress-induced membrane damage, but also due to internal processes executed in response to changing conditions and resulting in the cyst production (for example, in response to the nutrient depletion). We consider the second scenario more probable. It was demonstrated that tetracycline affects gene expression in eukaryotes affecting tetracyclinesensitive promoters and mitochondrial translation (Gossen and Bujard, 1992; Deuschle et al., 1995; Moullan et al., 2015; Sanchez et al., 2020). Therefore, this compound potentially can influence the physiology of dinoflagellates and intervene the initiation of intrinsically induced ecdysis.

In addition to such risks associated with antibiotics use in aquaculture, such as the resistance of bacteria and toxic effect of the applied compounds on cultured organisms, attention should be drawn to their impact on other components of ecosystems, such as dinoflagellates. While we showed that at the tested conditions tetracycline did not influence the level of ecdysis induced by mechanical stressors, it is still possible that this antibiotic can interfere with ecdysis triggered by other stressors. This question along with the investigation of mechanisms behind the tetracycline-dependent decrease in the natural level of ecdysis in the P. minimum culture are the agendas for future research.

\section{Acknowledgements}

The research was funded by the Russian Science Foundation, project No 18-74-10093.

\section{References}

Alderman D.J. and Hastings T.S. 1998. Antibiotic use in aquaculture: development of antibiotic resistance-potential for consumer health risks. Int. J. Food Sci. Tech. 33, 139-155.

Armstrong S.M., Hargrave B.T. and Haya K. 2005. Antibiotic use in finfish aquaculture: modes of action, environmental fate, and microbial resistance. In: Environmental effects of marine finfish aquaculture (Ed: Hargrave B.T.). Springer, Berlin, Heidelberg, pp. 341-357.

Berdalet E. 1992. Effects of turbulence on the marine dinoflagellate Gymnodinium nelsonii. J. Phycol. 28, 267-272.

Bostock J., McAndrew B., Richards R., Jauncey K., Telfer T., Lorenzen K., Little D., Ross L., Handisyde N., Gatward I. and Corner, R. 2010. Aquaculture: global status and trends. Philos. T. Roy. Soc. B. 365, 2897-2912.

Bravo I. and Figueroa R. I. 2014. Towards an ecological understanding of dinoflagellate cyst functions. Microorganisms. 2, 11-32.

Brown A.R., Lilley M., Shutler J., Lowe C., Artioli Y., Torres R., Berdalet E. and Tyler C.R. 2020. Assessing risks and mitigating impacts of harmful algal blooms on mariculture and marine fisheries. Rev. Aquacult. 12, 1663-1688.

Deuschle U., Meyer W.K.H. and Thiesen H.J. 1995. Tetracycline-reversible silencing of eukaryotic promoters. Mol. Cell. Biol. 15, 1907-1914.

Gossen M. and Bujard H. 1992. Tight control of gene expression in mammalian cells by tetracyclineresponsive promoters. Proc. Natl. Acad. Sci. U.S. A. $89,5547-5551$.

Guidi L.R., Santos F.A., Ribeiro A.C.S., Fernandes C., Silva L. H. and Gloria M.B.A. 2018. Quinolones and tetracyclines in aquaculture fish by a simple and rapid LC-MS/MS method. Food Chem. 245, 1232-1238.

Guillard R.R. and Ryther J.H. 1962. Studies of marine planktonic diatoms. I. Cyclotella nana Hustedt, and Detonula confervacea (Cleve) Gran. Can. J. Microbiol. 8, 229-239.

Hackett J.D., Anderson D.M., Erdner D. L. and Bhattacharya D. 2004. Dinoflagellates: a remarkable evolutionary experiment. Am. J. Bot. 91, 1523-1534.

Hallegraeff G.M. 1993. A review of harmful algal blooms and their apparent global increase. Phycologia. 32, 79-99.

Höhfeld I. and Melkonian M. 1992. Amphiesmal ultrastructure of dinoflagellates - a reevaluation of pellicle formation. J. Phycol. 28, 82-89.

Lafferty K.D., Harvell C.D., Conrad J.M., Friedman C.S., Kent M.L., Kuris A.M., Powell 
E.N., Rondeau D. and Saksida S.M. 2015. Infectious diseases affect marine fisheries and aquaculture economics. Annu. Rev. Mar. Sci. 7, 471-496.

Lulijwa R., Rupia E.J. and Alfaro A.C. 2020. Antibiotic use in aquaculture, policies and regulation, health and environmental risks: a review of the top 15 major producers. Rev. Aquacult. 12, 640-663.

Mallipattu S.K., Haidekker M., Von Dassow P., Latz M. and Frangos J. 2002. Evidence for shear-induced increase in membrane fluidity in the dinoflagellate Lingulodinium polyedrum. J. Comp. Physiol. A. 188, 409-416.

Matantseva O., Berdieva M., Kalinina V., Pozdnyakov I., Pechkovskaya S. and Skarlato S. 2020. Stressor-induced ecdysis and thecate cyst formation in the armoured dinoflagellates Prorocentrum cordatum. Sci. Rep. 10, 18322.

Matsuyama Y. 2012. Impacts of the harmful dinoflagellate Heterocapsa circularisquama bloom on shellfish aquaculture in Japan and some experimental studies on invertebrates. Harmful Algae. 14, 144-155.

Merino G., Barange M., Blanchard J.L., Harle J., Holmes R., Allison E.H., Badjeck M.K., Dulvy N.K., Holt J., Jennings S., Mullon C. and Rodwell L. 2012. Can marine fisheries and aquaculture meet fish demand from a growing human population in a changing climate? Glob. Environ. Change. 22, 795-806.

Morrill L.C. 1984. Ecdysis and the location of the plasma membrane in the dinoflagellate Heterocapsa niei. Protoplasma. 119, 8-20.

Morrill L.C. and Loeblich A.R. III. 1983. Ultrastructure of the dinoflagellate amphiesma. Int. Rev. Cytol. 82, 151-180.

Moullan N. Mouchiroud L., Wang X., Ryu D., Williams E.G. et al. 2015. Tetracyclines disturb mitochondrial function across eukaryotic models: a call for caution in biomedical research. Cell Rep. 10, 1681-1691.

Pollingher U. and Zemel E. 1981. In situ and experimental evidence of the influence of turbulence on cell division processes of Peridinium cinctum forma westii (Lemm.) Lefevre. Brit. Phycol. 16, 281-287.

Pozdnyakov I. and Skarlato S. 2012. Dinoflagellate amphiesma at different stages of the life cycle. Protistology. 7, 108-115.

Sanchez G., Linde S.C. and Coolon J.D. 2020. Genome-wide effect of tetracycline, doxycycline and 4-epidoxycycline on gene expression in Saccharomyces cerevisiae. Yeast. 37, 389-396.

Sapkota A., Sapkota A.R., Kucharski M., Burke J., McKenzie S., Walker P. and Lawrence R. 2008. Aquaculture practices and potential human health risks: current knowledge and future priorities. Environ. Int. 34, 1215-1226.

Shumway S.E. 1990. A review of the effects of algal blooms on shellfish and aquaculture. J. World Aquacult. Soc. 21, 65-104.

Smayda T. J. 2010. Adaptations and selection of harmful and other dinoflagellate species in upwelling systems. 2. Motility and migratory behaviour. Prog. Oceanogr. 85, 71-91.

Van de Waal D.B., Eberlein T., Bublitz Y., John U. and Rost B. 2014. Shake it easy: a gently mixed continuous culture system for dinoflagellates. J. Plankton Res. 36, 889-894.

Wang D.Z. 2008. Neurotoxins from marine dinoflagellates: a brief review. Mar. Drugs. 6, 349-371.

Wyatt T. and Jenkinson I. R. 1997. Notes on Alexandrium population dynamics. J. Plankton Res. 19, 551-575.

Address for correspondence: Mariia Berdieva. Institute of Cytology of the Russian Academy of Sciences, Laboratory of Cytology of Unicellular Organisms, Tikhoretsky Ave. 4, 194064 St. Petersburg, Russia; e-mail: maria.berd4@yandex.ru 